\title{
TORAL SUBGROUPS LYING IN THE CENTRALIZER OF THE GROUP OF UNITS
}

\author{
R. P. HUNTER
}

\begin{abstract}
Let $S$ be a compact connected finite dimensional monoid whose group of units $G$ is a compact connected Lie group. Then there is an open set $W$ about the unit element such that any compact subgroup within $W$ has dimension at most $\operatorname{dim} S-\operatorname{dim} G-1$ and if any toral subgroup achieves this dimension then that toral subgroup lies in the centralizer of $G$. Two applications are given, one to embeddings of irreducible monoids into $S$.
\end{abstract}

Let $G$ denote the group of units of a compact connected monoid, say, with zero, and let $Z(G, S)$ denote the centralizer of the group of units. The structure of $Z(G, S)$ is basically unknown. Indeed, it is an unsettled conjecture that it is connected [3]. For this aspect of $Z(G, S)$ see [2]. It is the purpose of this note to place certain elements and subgroups in $Z(G, S)$.

It will be shown that toral subgroups of maximal dimension sufficiently close to the unit element must lie in $Z(G, S)$. This will be used to show that certain irreducible monoids must also lie in $Z(G, S)$.

Let $G$ be a compact group which is the group of units of a finite dimensional compact connected monoid $S$. If $B$ is a closed subgroup of $S$ outside of the minimal ideal the product $G B$ can have dimension at most $\operatorname{dim} S-1$. (See [1].) If, in fact, one has $\operatorname{dim} G B=\operatorname{dim} S-1$ then $G B$ is a left group. Thus, if $B G$ also has dimension $\operatorname{dim} S-1$ one can conclude that $B$ meets $Z(G, S)=$ the centralizer of $G$. For this and related items see [4].

Now given any open set $V$ about the unit in a compact connected monoid there may exist nontrivial compact connected subgroups outside of the group of units. Indeed, using [1] and [3] one can show the following: Let $S$ be a compact connected monoid which is not a group. Suppose there is an open set $W$ in $S$ such that $W$ contains no connected subgroup outside of the group of units. Then there is a closed ideal $J$ such that $S / J$ contains a thread from zero to unit.

The following lemma is hardly unknown. It is stated for convenience. If $G$ is a transformation group of $X$, the stability subgroup at $x$ is denoted by $G_{x}$. In the following lemma there is no need to distinguish left and right stability.

LEMMA 1. Let $G$ be a connected group of units of a compact monoid and let $O$ be an open set about the unit. There exists about $G$ an open set $W$ such that $x \in W$ implies $G_{x} \subset O$. In particular, if $G$ is finite dimensional, there is about $G$ an open set $V$ such

Received by the editors April 14, 1978 and, in revised form, April 2, 1979.

AMS (MOS) subject classifications (1970). Primary 22A15; Secondary 22C05, 22 E20. 
that $x \in V$ implies $G_{x}$ is central and zero dimensional. If $G$ is a Lie group there is an open set $V$ about $G$ such that $G_{x}$ is trivial for $x \in V$.

Proof. Suppose, on the contrary, that $\left\{W_{\alpha}\right\}$ is a collection of open sets closing down on $G$ such that in each $W_{\alpha}$ there is an element $x_{\kappa} \in W_{\alpha}$ with $G_{x_{\alpha}}$ not contained in $O$. Let $h_{\alpha} \in G_{x_{\alpha}}$ be such that $h_{\alpha} x_{\alpha}=x_{\alpha}$. Let $\left\{x_{\alpha}\right\}$ cluster at $g$ and $h_{\alpha}$ cluster at $h \notin O$. Then $h g=g$ which is clearly impossible.

The remaining remarks follow from the structure theorem for finite dimensional compact connected groups and the fact that a Lie group does not have small subgroups.

The following definitions are convenient. Let $G$ be a group of units and $B$ a subgroup of a monoid $S$. Then

$$
\begin{aligned}
& Y(B, G)=\{g \mid g \in G, g B \subseteq B\}, \\
& Y(G, B)=\{g \mid g \in G, B g \subseteq B\} .
\end{aligned}
$$

Note that $g e \in B \Leftrightarrow g B=B \Leftrightarrow g B \subset B \Leftrightarrow g b \in B$ for some $b \in B$. If $S$ is compact and $G$ and $B$ are closed, then $Y(B, G)$ is a compact subgroup of $G$.

The following is proved in [1] and [4] with the help of a fibration

$$
Y(B, G) \rightarrow G \times B \rightarrow G B .
$$

LEMMA 2. Let $G$ be a finite dimensional compact group of units of a compact monoid $S$. There exists an open set $V$ about the identity such that both $Y(B, G)$ and $Y(G, B)$ are zero dimensional and central for any closed subgroup $B$ lying in $V$. If $S$ is also finite dimensional then the maximal dimension for a subgroup such as $B$ is $\operatorname{dim} S-\operatorname{dim} G-1$.

Finally, if $G$ is a connected Lie group the open set $V$ may be chosen so that any such Lie subgroup $B$ having maximum dimension must meet $Z(G, S)$.

In the study of monoids whose groups of units have low complementary dimension (or codimension) a critical fact has been the following: If $G$ is a compact connected Lie group and $H$ is a closed subgroup such that $G / H$ is one dimensional then $H$ is normal [3].

The following is a natural extension of this.

LEMMA 3. Let $G$ be a compact connected Lie group and $H$ a closed subgroup of $G$ such that $G / H$ is topologically a torus. Then $H$ is normal.

Proof. The group $G$ acts upon $G / H$ in the usual way. If $F$ denotes the normal subgroup $\cap\left\{g \mathrm{Hg}^{-1}: \mathrm{g} \in G\right\}$, consisting of those elements of $G$ fixing all elements of $G / H$, then $G / F$ acts effectively and transitively. It follows from [8] that $G / F$ is a toral group. This means that $F$ contains the semisimple part of $G$. Since $F$ is contained in $H$ and since any subgroup containing the semisimple part is normal, $H$ must be normal.

The above lemma will be crucial in what follows. The following lemma is somewhat convenient. 
LEMMA 4. Let $G$ be a compact connected Lie group of units and let $B$ be a toral subgroup of a compact monoid $S$. If $G B$ is a subgroup then $G e$ is a normal subgroup of $G B$, where $e^{2}=e \in B$. Thus, there is a short exact sequence

$$
1 \rightarrow G e \cap B \stackrel{\Delta}{\rightarrow} G e \text { (s) } B \stackrel{m}{\rightarrow} G B \rightarrow 1
$$

where $\Delta(b)=\left(b^{-1}, b\right)$ and $m(t, b)=t b$.

In particular, if $G e \cap B=\{e\}$ then $G B$ is the direct product of $G e$ and $B$.

Proof. Suppose $G B$ is a group. Then $G e$ is a subgroup of $G B$ since $g \mapsto g e$ is a homomorphism on $G$. The orbit space of $G B$ under the action of $G e$, on the left, is a homogeneous space of $B$. But the last must be a torus. Thus, by Lemma 3, Ge is normal. The rest of the lemma is a simple calculation.

LEMMA 5. Let $S$ be a compact connected finite dimensional monoid whose group of units is a compact connected Lie group. Then there exists an open set $W$ about the unit element such that (1) for each $x \in W$ the stability groups both left and right are trivial;

$$
\{g \in G: g x=x\}=\{g \in G: x g=x\}=\{1\} .
$$

(2) If $B$ is any closed subgroup contained in $W$ then $\{g \in G:$ ge $\in B\}$ is trivial where $e^{2}=e \in B$, i.e. $G e \cap B=\{e\}$.

Proof. This can be established as in [1]. For convenience, we include an argument for (2). Let $B_{\alpha}$ having units $e_{\alpha}$ be a net of closed subgroups converging to $\{1\}$ such that $G_{\alpha}=\left\{g \in G: g e \in B_{\alpha}\right\}$ is nondegenerate. Since $G$ is a Lie group there is an open set $V$ such that $G_{\alpha} \backslash V$ is always nonempty. Pick $g_{\alpha}$ in $G_{\alpha} \backslash V$. Then $g_{\alpha}$ clusters to some $g \notin V$. However, we would then have $g_{\alpha} e_{\alpha}$ clustering to $g$ but converging to 1 .

Proposition 1. Let $S$ be a compact connected finite dimensional monoid whose group of units $G$ is a compact connected Lie group. Then there is an open set $W$ about the unit element such that the following hold.

(1) Any compact connected subgroup of $S$ lying in $W$ has dimension at most $\operatorname{dim} S-\operatorname{dim} G-1$. Any such subgroup must meet $Z(G, S)$. (If $G$ is semisimple the subgroup need only have dimension $\operatorname{dim} S-\operatorname{dim} G-2$ to conclude that it meets $Z(G, S)$.)

(2) Any toral subgroup having dimension $\operatorname{dim} S-\operatorname{dim} G-1$ and lying in $W$ lies entirely in $Z(G, S)$. In either case $G B$ coincides with the component of $e$ in $H_{e}$. In the second case $W$ may be chosen so that $G B$ is isomorphic to $G \times B \cong G e \times B$ under $g b \leftrightarrow(g e, b)$.

Proof. Choose $W$ small enough so that all stability groups are trivial and for any subgroup $B \subseteq W$ the set $\{g \in G: g e \in B\}$ where $e^{2}=e \in B$ is trivial. This is possible by the previous lemmas. Then statement (1) follows directly from [4].

To prove (2) note first that if $B$ is any toral group lying in $W$ then $G B$ is the identity component of $e$ in $H_{e}$ where $e^{2}=e \in B$. This follows directly from [1] and [4] since $\operatorname{dim} G B=\operatorname{dim} G+\operatorname{dim} B=\operatorname{dim} S-1$. The idempotent $e$ lies in $Z(G, S)$ so that $G e$ is a subgroup of $G B$. Moreover, $G e$ is normal in $G B$ by Lemma 
2. Thus $B$ acts upon $G e$ by inner automorphisms. But $G$ and $G e$ are isomorphic under the isomorphism $g \mapsto g e$ since this mapping is a homomorphism and $e \in W$. The idea of the argument is that as the subgroups $B$ approach $\{1\}$ the action of the inner automorphisms must become trivial since $\operatorname{Aut}(G)$ is a Lie group.

In effect, let $M$ be a compact neighborhood of $\{1\}$, contained in $W$.

Let $\tilde{E}$ denote those idempotents in $M$ which are also in the centralizer of $G$. Thus $\tilde{E}=E \cap Z(G, S) \cap M$. Then $\tilde{E}$ is a compact set and multiplication $m$ cut down to $G \times \tilde{E}$ is a homeomorphism. Indeed if $g_{1}, g_{2} \in G, e_{1}, e_{2} \in \tilde{E}$ and $g_{1} e_{1}=g_{2} e_{2}$ we first note that $g_{i} e_{i}$ lies in the maximal subgroup determined by $e_{i}, i=1,2$, since multiplication by $e_{i}$ is a homomorphism on $G$. Thus $e_{1}=e_{2}$. But then $g_{1}=g_{2}$ since $e_{1}=e_{2}$ is inside of $W$.

Next, let $\tau$ denote the union of all those toral subgroups $T$ contained in $M$ such that $T$ meets the centralizer of $G$ and $G T$ is a subgroup of $S$. (We know that $T \subset \tau$ if, for example, $\operatorname{dim} T=\operatorname{dim} S-\operatorname{dim} G-1$. This again is from [4].) If $e^{2}=e \in$ $T \subset \tau$, we know from Lemma 4 that $T$ acts upon $G e$ via inner automorphism: $g e \mapsto t(g e) t^{-1}$.

We now define the action of $\tau$ upon $G$ through the diagram:

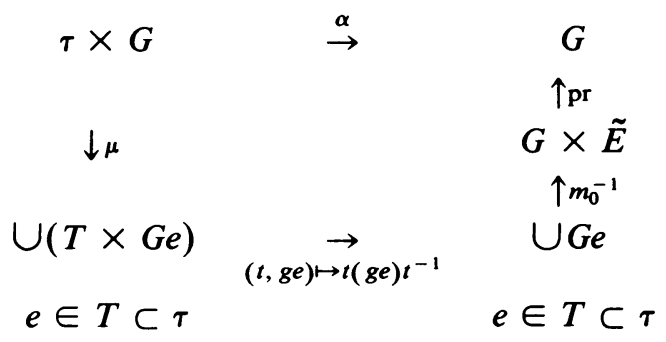

Here $\mu(t, g)=(t, g e)$ where $t \in T$ and $e$ is the unit of $T$. From the fact that $\mathcal{H}$ is upper semicontinuous $\mu$ is continuous. The inverse of the multiplication map $G \times \tilde{E} \rightarrow G \tilde{E}$ cut down to $\cup G e, e \in T \subset \tau$, is denoted by $m_{0}^{-1}$.

Thus, the above diagram consists of continuous functions. We have then, a continuous function $\varphi: \tau \mapsto \operatorname{Aut}(G), \varphi(t)=\alpha(t, g)$.

Using the compact open topology $\operatorname{Aut}(G)$ is a Lie group. In particular, there is a neighborhood $V$ of $\{1\}$ containing only trivial subgroups. Thus, $\varphi^{-1}(V)$ is a neighborhood of $\{1\}$ in $\tau$. For each $T \subset \varphi^{-1}(V)$ we must have $\varphi(T)=\{1\}$.

By a definition of $\varphi$ this means simply that $T$ lies in $Z(G, S)$.

Recall that the rank of a compact Lie group is defined as the dimension of a maximal torus. We denote it by rk $G$.

Proposition 2. Let $S$ be a compact connected monoid of finite dimension whose group of units $G$ is a connected Lie group. Then there is an open set $O$ about $G$ such that any toral subgroup lying in $O$ satisfies $\operatorname{dim} T<\operatorname{dim} S-\operatorname{dim} G+\mathrm{rk} G$.

Moreover, if $T$ is normal in its maximal subgroup then $\operatorname{dim} T=\operatorname{dim} S-\operatorname{dim} G$ + rk $G-1$ implies that $T$ meets $Z(G, S)$.

If $G$ is semisimple and $T$ is normal in its maximal subgroup then $\operatorname{dim} T>\operatorname{dim} S$ $-\operatorname{dim} G+\mathrm{rk} G-2$ implies that $T$ meets $Z(G, S)$. 
Proof. Let $O$ be chosen to miss the minimal ideal and so that left and right stability groups at any $x \in O$ are trivial. We refer here to Lemma 1 . Thus $g x=x$ implies $g=1$ and $x g=x$ implies $g=1$.

Since $T G$ is a manifold we must have $\operatorname{dim} S>\operatorname{dim} T G$. Moreover $\operatorname{dim} T G=$ $\operatorname{dim} T+\operatorname{dim} G-p$ where $p$ is the dimension of the stability group $(T \times G)_{e}$. (The idempotent of $T$ is denoted by $e$.) This subgroup is the set of all $(t, g)$ such that $t=e g$. Since $e g=e g^{\prime} \Rightarrow g=g^{\prime}$ it follows that $(T \times G)_{e}$ is isomorphic with $\{g \in g G$, eg $\in T\}=W$. Since $T$ is abelian and $w \rightarrow e w$ is an isomorphism, $W$ is abelian so that $\operatorname{dim} W \leqslant \mathrm{rk} G$. Hence

$$
\operatorname{dim} S>\operatorname{dim} T+\operatorname{dim} G-\operatorname{dim} W>\operatorname{dim} T+\operatorname{dim} G-\mathrm{rk} G .
$$

This establishes the first assertion.

Next suppose that $T$ is normal in its maximal subgroup. In this case, [4], GTG is the total space of a fiber bundle over a certain homogeneous space $G / Y$. Indeed, $Y$ is the subgroup of $G$ consisting of those $g$ with $g T G \subseteq T G$. The fiber of the fiber space $G T G \rightarrow G / Y$ is $T G$. Thus, from [4] and the above,

$$
\begin{aligned}
\operatorname{dim} S & >\operatorname{dim} G T G=\operatorname{dim} T G+\operatorname{dim} G / Y \\
& \geqslant \operatorname{dim} T+\operatorname{dim} G-\mathrm{rk} G+\operatorname{dim} G / Y .
\end{aligned}
$$

The condition $\operatorname{dim} T \geqslant \operatorname{dim} S-\operatorname{dim} G+\mathrm{rk} G-1$ means that $\operatorname{dim} G / Y=0$. This shows that $G e \subseteq H_{e}$. In the same way, one concludes that $e G \subseteq H_{e}$. This places $e$ in $Z(G, S)$.

For the final assertation one argues as above and uses the fact mentioned before that the dimension of a homogeneous space of a compact connected semisimple Lie group cannot be one.

Following is a remark on the condition that a subgroup $B$ be normal in its maximal subgroup:

This is one possible condition upon a subgroup $B$ so that a fibering $G B G \rightarrow$ $G / Y$ is possible. Thus if $B$ is normal in its maximal subgroup then for $g \in G$, $g B G \cap B G \neq \square$ implies $g B G=B G$ and this is the condition needed. (See [4].)

The following example, perhaps the simplest possible, shows that if $B$ is not normal in its maximal subgroup this condition need not hold.

The kernel of $S$ will be $S_{3}$ viewed, say, as $\left\langle a, b \mid a^{2}=e=b^{3}, a b a=b^{2}\right\rangle$. The group of units will be $\left\langle\bar{a} \mid \bar{a}^{2}=\bar{e}\right\rangle=Z_{2}$. Let $S$ be the disjoint union of $S_{3}$ and $Z_{2}$ with the multiplication completed by $\bar{a} e=e \bar{a}=a$ and $\bar{e} e=e \bar{e}=e$. This is simply the direct product of $\{0\} \cup\{1\}$ and $S_{3}$ with the complement of $\{1\} \times\{a, e\}$ in $\{1\} \times S_{3}$ deleted.

Now let $B=\{b a, e\}, G=\{\bar{a}, \bar{e}\}$. One finds that $B G=\{b a, a, b, e\}$ while $\bar{a} B G=\left\{b^{2}, e, a b, a\right\}$. Thus $\bar{a} B G \cap B G=\{a, e\}$.

We now give an application of Proposition 1 to the centralizer conjecture. Throughout the rest of this paper basic familiarity with the theory of irreducible monoids will be assumed.

It is shown in [1] that if $A$ is irreducible and is embedded in $S$, a compact connected finite dimensional monoid, then an upper bound for the dimension of subgroups of $A$ near the identity is $\operatorname{dim} S-\operatorname{dim} G-1$. Thus, if this upper bound 
is attained by Lie groups, i.e. toral groups since $A$ is abelian, then, sufficiently close to the identity, idempotents of $A$ lying in such subgroups also lie in $Z(G, S)$.

Proposition 3. Let $A$ be an algebraically irreducible monoid with nondegenerate subgroups near the identity and suppose that all maximal subgroups not containing the identity are toral subgroups of dimension $k$. (Note that $A$ is necessarily of dimension $k+1$.) Let $S$ be a finite dimensional compact connected monoid and let $G$, its group of units, be a Lie group. Suppose that $A$ can be embedded as a submonoid of $S$. Then $k<\operatorname{dim} S-\operatorname{dim} G$. If $k=\operatorname{dim} S-\operatorname{dim} G-1$ then there is an open set $V$ in $A$ about the identity which is contained in $Z(G, S)$.

Proof. There exists an open set $O$ about the identity in $S$ such that any toral subgroup $t$ of $\operatorname{dimension} \operatorname{dim} S-\operatorname{dim} G-1$ lies in $Z(G, S)$ so that $G T=T G$ is isomorphic to the direct product $G \times T \cong G e \times T$. Thus, there is an idempotent $q$ of $A$ such that any maximal subgroup of $A$ above $q$ behaves as $T$ above. Hence, without loss of generality, we may assume that all maximal subgroups of $A$ behave as $T$, that is to say, lie in $Z(G, S)$ and form direct products with $G$. Now consider two idempotents $e$ and $f$ in $A$ with $e<f$ and no idempotents of $A$ between $f$ and $e$. Let $F$ denote the union of all $H$-classes of $A$ between and including those of $e$ and $f$. Then $F$ is a compact connected monoid with group of units say $U$ which is the $H$-class of $f$ in $A$, and minimal ideal $K$ which is the $H$-class in $A$ of $e$. Next let $X$ denote the compact connected monoid generated by $G$ and $F$. It is now claimed that the group of units of $X$ is $G U$ and that the minimal ideal of $X$ is the group $G K$. This requires the following two lemmas.

LEMMA 6. Let $X$ a be compact connected monoid and $F$ a compact connected submonoid of $X$. Suppose that $H$ is a compact connected subgroup of the group of units and that $H$ contains the group of units of $F$. If $X$ is the closure of the submonoid generated by $H$ and $F$ then the group of units of $X$ is connected.

Proof. Suppose, on the contrary, that $Q$, the group of units, is not connected. Then there are disjoint open sets $V, W$ such that $C$, the component of 1 in $Q$, is contained in $V$ and $Q$ meets $W$. By the continuity of multiplication there is an open set $O$ about $C$ such that $O^{2} \subset V$. Now let $J$ be the closed ideal generated by the closed set $X \backslash(O \cup W)$. We may then write $X \backslash J=W^{\prime} \cup O^{\prime}$ where $W^{\prime} \subset W$ and $O^{\prime} \subset O$. Moreover $W^{\prime}$ contains no elements of $H \cup F$. It is now claimed that no product $m=m_{1} \cdot m_{2} \cdots \cdot m_{j}$ can be in $W^{\prime}$ when $m_{i} \in H \cup F, 1<i<j$. Suppose, on the contrary that this product is in $W^{\prime}$ and $j$ is chosen minimal. Then $m_{1} \cdot m_{2} \cdots \cdot m_{j-1}$ is in either $J$ or $O^{\prime}$. If it is in the ideal $J$ then $m$ must be in $J$. Thus, we may assume $m_{1} \cdot m_{2} \cdots \cdot m_{j-1} \in O^{\prime}$. Now if $m_{j} \in J$ we again have $m \in J$ and if $m_{j} \in O^{\prime}$ we would have $m \in O^{\prime} m_{j} \subset O^{2} \subset V$. In any case we would have a contradiction. Thus $Q$ is connected.

LeMma 7. Let $X$ be a compact monoid with group of units $H$. Let $F$ be a compact submonoid whose minimal ideal $K$ is a subgroup such that $H K=K H$. If $X$ is the closure of the submonoid generated by $H$ and $F$ then the minimal ideal of $X$ is the subgroup $H K$. 
Proof. If $h \in H, k \in K$ we note that $h k H K=h(k H) K=h H \bar{k} k=H K$ and that $H K h k=H K$ so that $H K=K H$ is a subgroup. Moreover $H K$ is an ideal in the submonoid generated by $H \cup F$ since $F(K H) \subseteq K H$ and $(K H) F=H K F \subset H K$, etc. By continuity $H K$ is an ideal in the submonoid generated by $H \cup F$. But an ideal which is a subgroup is always a minimal ideal.

We return to the argument. From Lemma 6 we see that $G U$ is contained in the group of units of $X$ and that the latter is connected. But $G U$ is connected and has dimension $\operatorname{dim} S-1$. Clearly then both $G U$ and the group of units of $X$ are both equal to the component of $f$ in $H_{f}$.

It follows that in a neighborhood of $G U$ the natural mapping $\gamma: X \rightarrow X / G U$ is a homomorphism onto a one dimensional monoid. In effect, $X$ contains a closed ideal $J$ such that the orbits of $G U$ on $X / J$ form a quotient space which is necessarily one dimensional since $\operatorname{dim} G U=\operatorname{dim} X-1$ and $G U$ is a Lie group. When such a quotient is one dimensional the natural mapping is a homomorphism [6]. Thus $\tilde{X}$, the quotient space of $X / J$ under the orbits of $G U$ is a one dimensional monoid. Then, [7], there is a standard thread from zero to identity in $\tilde{X}$. Going from $X$ to $\tilde{X}$ we see that $F$ must map onto this standard thread. Since $G$ and $F$ generate $X$ it follows that, in fact, $\tilde{X}$ is a standard thread.

Now denote $G U$ by $H$, further $G f$ by $L$, and $G K$ by $M$. Application of the next lemma produces a connected subsemigroup joining $f$ and $e$, lying in $Z(G, S)$ and lying in $F \subset A$. From the irreducibility of $A$ we see that $A \subset Z(G, S)$.

LEMMA 8. Let $X$ be a compact connected monoid having a minimal ideal $M$ which is a group with identity $e$. Suppose that $X$ contains a closed ideal $J$ such that the (left) orbits of $H$, the group of units, defines a homomorphism of $X / H$ onto a standard thread.

Suppose that $F$ is a compact connected submonoid of $X$ containing just two idempotents. Denote the group of units of $F$ by $U$ and denote the minimal ideal of $F$ by $K$. Suppose next that $U$ lies in the center of $H, K$ lies in the center of $M$ and that there is a closed subgroup $L$ of $H$ such that $H=L U$ and $x \rightarrow x e$ defines $a$ monomorphism of $L$ into $M$.

If all these things are fulfilled, then $F$ contains a compact connected submonoid which contains $e$ and lies in $Z(H, X)$.

Proof. Making the usual identifications let $\gamma: X / J \rightarrow[0,1]$ be the orbit homomorphism defined by $H$. From [3] we know that there is a local thread $P$ lying in $Z(H, X / J)$. Thus, by making $J$ larger if necessary we may assume that $\gamma(P)=$ $[0,1]$. Clearly $\gamma(F / F \cap J)=[0,1]$. Thus, given $a \in F \backslash J$ there is an element $h \in H$ such that $h p=a$ for some $p$ with $\gamma(p)=\mu(a)$. We claim that $h$ must lie in the center of $H$. Note that $h$ can be written as $h=q u$ where $q \in L$ and $u \in U$. Thus qup $=a$. Then $(q e)(u e)(p e)=a e$ or $q e=(a e)(p e)^{-1}(u e)^{-1}$. We note that ae is in the center of $M$ since $a e \in K$. Next, $(p e)$ lies in the centralizer of $L e$ in $M$ since $p r=r p$ for any $r$ in $H$. Finally (ue) lies in the centralizer of $L e$ since $u \in U$. Thus qe lies in the centralizer of $L e$ in $M$. In other words, $q e$ lies in the center of Le. But $x \rightarrow x e$ being a monomorphism puts $q$ in the center of $L$. Thus $L=q u$ is 
in the center of $H$. Since $h p=a$ it follows that $a$ lies in the centralizer of $H$. Thus $F \backslash J \subseteq Z(H, X)$. The remainder is now standard. The monoid $F / F \cap J$ contains a thread from identity to zero, which, from the above, lies in $Z(H, X)$. Since $F$ has just two idempotents it now follows that $F$ contains the desired submonoid.

Corollary. Let all things be as in Proposition 3. Then there is a closed ideal $J$ such that $A / J$ lies in $Z(G, S / J)$. Thus $Z(G, S / J)$ is connected. Moreover $\operatorname{dim} Z(G, S / J)=\operatorname{dim} S$.

One difficulty encountered with irreducible semigroups is that they may enter and leave $Z(G, S)$ infinitely often as they approach the unit element.

EXAMPLE. There exists a compact connected monoid $S$ containing a standard thread $I$ from zero to unit element such that:

(1) $E \cap I=Z(G, S) \cap I$ is a sequence of idempotents, $\left\{e_{i}\right\}$ converging to $\{1\}$ and

(2) each interval $\left[e_{i}, e_{i+1}\right]$ is a nil thread.

We indicate the construction which uses some standard devices ([3], [5]).

Let $\boldsymbol{H}$ denote a compact connected semisimple Lie group with trivial center and let $J$ denote the usual nil interval. In $H \times J$ consider $T \times J$ where $T$ is a circle subgroup of $H$. Now $T \times J$ contains a one-parameter subsemigroup $P$ such that $P$ meets the centralizer of $H$ at only the unit and the idempotent in the minimal ideal. (Thus, $P$ winds one time, and $P / T$ is a nil thread.)

Let $I$ be the unit interval with each $n /(n+1), n=0,1,2, \ldots$, and $\{1\}$ idempotent. Each interval between two idempotents is taken as a nil thread. For each $i$ take $H_{i}$ as a copy of, say, $S O(3)$ and let $G=\times H_{i}, i=0,1,2, \ldots$ Now form $G \times I$ and denote its idempotents $(1, n /(n+1))$ by $e_{n}$. For each $i$ locate $H_{i} \times[i /(i+1),(i+1) /(i+2)]$. For $T_{i}$ a circle subgroup of $H_{i}$ build a one parameter subsemigroup $P_{i}$ such as the one above. The $P_{i}$ thus constructed meets $Z(G, S)$ at only $e_{i}$ and $e_{i+1}$. Upon $G \times I$ we define a congruence $\sim$ as follows. On the subsemigroup $G \times[0, i /(i+1)]$ the classes of $\sim$ are the orbits of the group $\tilde{G}_{i}=H_{i} \times H_{i+1} \times H_{i+2} \times \ldots$ (All coordinates before $i$ are set equal to $\{1\}$.)

In each case, the minimal ideal of $P_{i}$ lies in a single class of $\sim$. Indeed $P_{i} / \sim$ is always a nil thread and $P_{i} / \sim$ meets $Z(G, S / \sim)$ at only $e_{i+1}$ and $e_{i}$. Note that $G$ is left unchanged by $\sim$ and is the set of points at which $S / \sim$ fails to be finite dimensional. The desired thread is the union of the $P_{i} / \sim$.

\section{REFERENCES}

1. L. W. Anderson and R. P. Hunter, A remark on finite dimensional compact connected monoids, Proc. Amer. Math. Soc. 42 (1974), 602-606.

2. K. H. Hofmann and R. P. Hunter, On a centralizer result of Hunter, Semigroup Forum 6 (1973), 365-372.

3. K. H. Hofmann and P. S. Mostert, Elements of compact semigroups, Merrill, Columbus, Ohio, 1966.

4. R. P. Hunter, Certain idempotents lying in the centralizer of the group of units, Proc. Amer. Math. Soc. 71 (1978), 339-344. 
5. On the structure of homogroups and their applications to compact connected semigroups, Fund. Math. 52 (1963), 62-102.

6. R. P. Hunter and L. W. Anderson, Compact semigroups having certain one dimensional hyperspaces, Amer. J. Math. 92 (1970), 894-896.

7. R. P. Hunter, On one dimensional semigroups, Math. Ann. 146 (1962), 383-396.

8. D. Montgomery and H. Samelson, Groups transitive on the n-dimensional torus, Bull. Amer. Math. Soc. 49 (1943), $455-456$.

Department of Mathematics, The Pennsylvania State University,University Park, PennsylVANIA 16802 